\title{
Validation and Application of a Computational Model for Wrist and Hand Movements Using Surface Markers
}

\author{
Cheryl D. Metcalf*, Member, IEEE, Scott V. Notley, Paul H. Chappell, Member, IEEE, Jane H. Burridge, and \\ Victoria T. Yule
}

\begin{abstract}
A kinematic model is presented based on surface marker placement generating wrist, metacarpal arch, fingers and thumb movements. Standard calculations are used throughout the model and then applied to the specified marker placement. A static trial involving eight unimpaired participants was carried out to assess inter-rater reliability. The standard deviations across the data were comparable to manual goniometers. In addition, a test-retest trial of ten unimpaired participants is also reported to illustrate the variability of movement at the wrist joint, metacarpal arch, and index finger as an example of model output when repeating the same task many times. Light and heavyweight versions of the tasks are assessed and characteristics of individual movement strategies presented. The participant trial showed moderate correlation in radial/ulnar deviation of the wrist ( $r=0.65$ ), and strong correlation in both metacarpal arch joints $(r=0.75$ and $r=0.85)$, the MCP $(r=0.79)$, and PIP $(r=0.87)$ joints of the index finger. The results indicate that individuals use repeated strategies of movement when lifting light and heavyweight versions of the same object, but showed no obvious repeated pattern of movement across the population.
\end{abstract}

Index Terms-Biomedical measurements, hands, kinematics, motion analysis.

\section{INTRODUCTION}

$\mathbf{K}$ INEMATIC investigations of functional movements are increasingly required in clinical research to further understand the appropriateness of rehabilitation techniques and to quantify the effectiveness of such techniques [1]. Historically, this has been well developed in gait analysis but the added complexity of functional movements available in the upper limb has hindered assessment especially in a clinical research setting, which relies on simplified techniques and ease of application to minimize the impact on the patient.

Manuscript received August 18, 2006; revised July 26, 2007. This work was supported in part by the University of Southampton's Life Sciences Interfaces Forum. Asterisk indicates corresponding author.

${ }^{*}$ C. D. Metcalf is with the School of Electronics and Computer Science, Room 3231, Building 59, University of Southampton, Southampton SO17 1BJ, U.K. (e-mail: cdm@ecs.soton.ac.uk).

S. V. Notley is with the Institute of Sound and Vibration Research, University of Southampton, Southampton SO17 1BJ, U.K.

P. H. Chappell is with the School of Electronics and Computer Science, University of Southampton, Southampton SO17 1BJ, U.K.

J. H. Burridge and V. T. Yule are with the School of Health Professions and Rehabilitation Sciences, University of Southampton, Southampton SO17 1BJ, U.K.

Digital Object Identifier 10.1109/TBME.2007.908087
Hand movement and upper limb movement, in general, is complex [2]. The wrist is often defined as having two degrees of freedom: flexion/extension and radial/ulnar deviation. The movements of the hand, fingers, and thumb provide the functional ability that is required for prehension. The hand, in terms of the palm, can flex and extend to facilitate the opposition of the thumb to the fingertips. The fingers themselves are also capable of multiple degrees of freedom including flexion/extension of the metacarpophalangeal, proximal and distal interphalangeal joints, as well as abduction/adduction at the metacarpophalangeal joints. There is also a small amount of rotation at the fingers. The thumb, which is often ignored in most models of hand movement, is capable of abduction from the palm, rotation through to opposition, and flexion/extension of the metacarpophalangeal and interphalangeal joints.

Various models have been developed which attempt to characterize all the complex movements of the hand [3]-[7], whereas others are only concerned with specific aspects such as joint rotations or wrist angles [8]-[11]. The work of Degeorges et al. [10] is concerned with only modeling 3-D rotations of the index finger joints. Their protocol uses a system of markers placed at the end of rods in triads to denote the axis for each segment. Although they only analyze the movement of a single finger, their method requires the use of a thermoplastic cast molded for each participant and they conclude that a major difficulty of the system was the number of markers in a small sized capture volume. Fowler et al. [9] use a similar system of rod axis markers arranged in triads for the functional and biomechanical assessment of normal and rheumatoid hand. As an indication of the complexity of this method 12 , markers are used just to analyze flexion/extension and rotation of the metacarpalphalangeal joint.

With surface markers, skin movement may be a problem for accurate measurement of angles and joint segments [8], [12]. The work of Schmidt et al. [13] concludes that it is necessary to consider skin movement for accurate measurement of wrist angles. They developed a rigid-segment marker placement method to minimize skin movement based on markers placed in triads. Su et al. [4] attempt to account for skin movement by using two markers per segment of the fingers placed proximally and distally to each joint. A similar system with two markers per segment is used by Ryu et al. [8] to analyze the relationship between the surface marker placements and the underlying anatomy by comparing MRI images with model outputs for static hand positions. 
Miyata et al. [3] have developed a computational model to generate joint angles of the wrist, hand, fingers, and thumb using single markers per joint. They discuss the advantages and disadvantages of using complex protocols with large numbers of markers to model all degrees of freedom of the hand. The model subsequently developed models less degrees of freedom of the hand and uses a total of 25 surface markers. Similarly, Carpinella et al. [5] developed a model using a small number of markers to calculate finger and thumb movements using a single surface marker placed above each joint axis. The two methods are comparable for generating finger and thumb movements although the method of Carpinella et al. [5] does not include movements of the wrist.

Small et al. [6] developed a model of wrist movement using only six single surface markers and as such was suitable in clinical applications. Their method relied heavily on the underlying anatomy for precise marker placement and concludes that radiographic methods to measure motion are no more accurate than surface measurements. Murgia et al. [14] also concentrated only on the analysis of wrist movement using single surface markers. The model requires seven markers placed on bony landmarks on the elbow, wrist and hand. The problem with using such a wide surface area when applying markers is the possibility of marker occlusion for markers more proximal to the body.

From the literature, it may been seen that no standardized method of applying markers to the upper limb exists as there does for lower limb analysis [1] and although standards have been proposed [15] they are based on boney anatomical landmarks beyond those obtainable directly from surface markers [1] and are often too time consuming and laborious to apply in a patient trial.

As an example, four commonly used marker placement methods to gather information regarding the movement of the proximal interphalangeal joint of the second finger are:

1) three linear markers placed in the metacarpophalangeal, proximal, and distal interphalangeal joints [5], [15];

2) two markers placed at the distal and proximal heads of the proximal and medial phalanges of the second finger [4], [16] (four markers in total);

3 ) three noncollinear markers forming a triangle placed on both the proximal and medial phalanges of the second finger [3] (six markers in total);

4) a rod axis system consisting of three or more noncollinear markers on a frame above the joint or phalanx [9], [10].

Marker placement methods $2-4$ are useful in a biomechanical research setting where the objective is to minimize as much as possible the effects of skin movement on an identified segment. Marker placement method 1 is often used in a clinical research setting where the emphasis is on clearly identifiable and repeatable placement using palpation techniques and where the effect on the patient is kept to a minimum. Simple marker placements can also be applicable to research involving children, where the surface area of the hands are significantly smaller than would be required by marker placement systems as described in methods 3 and 4.

This paper outlines the method used to develop a protocol for modeling wrist, hand, finger, and thumb movements with an emphasis on clearly identifiable and repeatable marker placements
TABLE I

MARKER LABELS AND POSITIONS FOR 3-mm HEMISPHERICAL MARKERS

\begin{tabular}{ll}
\hline \hline Marker & Description of Placement \\
WRU & Distal head of the ulnar \\
WRR & Distal head of the radial styloid process \\
FAU & Dorsal aspect of the ulnar \\
FAR & Dorsal aspect of the radius \\
CMC1 & Proximal head of the 1st metacarpal at the carpometacarpal joint \\
CMC2 & Proximal head of the 2nd metacarpal at the carpometacarpal joint \\
CMC5 & Proximal head of the 5th metacarpal at the carpometacarpal joint \\
MCP1 & Distal head of the 1st metacarpal \\
MCP2 & Distal head of the 2nd metacarpal \\
MCP3 & Distal head of the 3rd metacarpal \\
MCP4 & Distal head of the 4th metacarpal \\
MCP5 & Distal head of the 5th metacarpal \\
IP & Distal head of the proximal phalanx of the thumb \\
FT1 & Distal head of the distal phalanx of the thumb \\
PIP2 & Distal head of the proximal phalanx of the second finger \\
DIP2 & Distal head of the medial phalanx of the second finger \\
FT2 & Distal head of the distal phalanx of the second finger \\
PIP3 & Distal head of the proximal phalanx of the third finger \\
DIP3 & Distal head of the medial phalanx of the third finger \\
FT3 & Distal head of the distal phalanx of the third finger \\
PIP4 & Distal head of the proximal phalanx of the fourth finger \\
DIP4 & Distal head of the medial phalanx of the fourth finger \\
FT4 & Distal head of the distal phalanx of the fourth finger \\
PIP5 & Distal head of the proximal phalanx of the fifth finger \\
DIP5 & Distal head of the medial phalanx of the fifth finger \\
FT5 & Distal head of the distal phalanx of the fifth finger \\
CMCVM & Virtual marker created halfway between CMC2 and CMC5 \\
\hline \hline
\end{tabular}

suitable for clinical applications. As such, the protocol identifies specific single surface marker placements which can be easily identifiable through palpation techniques, and which will not interfere with a participants' normal range of movement or compensatory movement strategies and is quick to apply. This method could also be applied to studies involving children or patient groups. The proposed model generates vectors and planes based on those marker placements. A validation procedure is undertaken analyzing the variances between the model output and known angle measurements of the wrist. The inter-rater reliability of surface marker placements is assessed. The model is then applied to a participant repeatability trial to analyze the movement strategies of individuals when repeating light and heavy versions of the same lifting task. This task enables a subjective assessment of the model in a practical setting with each joint moving in all degrees of freedom. An analysis is undertaken to ascertain whether there is any correlation to be found in movement strategies for an individual and across a population.

\section{METHODS}

\section{A. System and Setup}

A Vicon 460 6-camera movement analysis system (Vicon, Oxford, UK) sampling at $100 \mathrm{~Hz}$ was used to capture data for all the trials. Twenty-six passive reflective markers were placed at specific positions on the wrist and hand. Table I illustrates the marker placements for the $26 \times 3 \mathrm{~mm}$ hemispherical markers. Particular attention was needed when choosing marker positions, especially those placed on the wrist and fingers. From the previously outlined marker placement methods, an altered version of the first option was used in this instance as it maintained the integrity of the movement, i.e., not impeding the natural movement of the joints. Marker placements were selected 


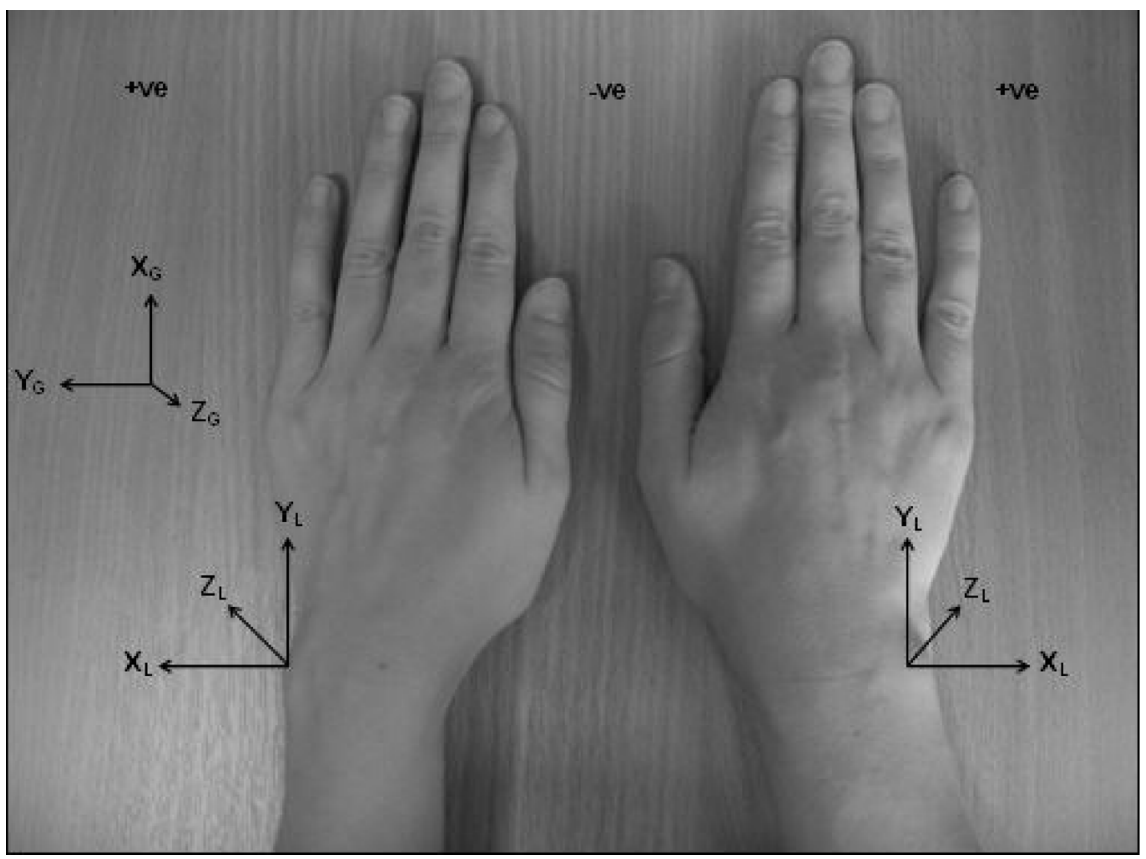

Fig. 1. Local coordinate system $\left(\mathrm{X}_{\mathrm{L}}, \mathrm{Y}_{\mathrm{L}}, \mathrm{Z}_{\mathrm{L}}\right)$ as defined by the model. The definition of wrist movement is presented, whereby radial deviation $=-$ ve and ulnar deviation $=+v e$; flexion is defined as +ve and extension $=-$ ve. Radial/ulnar deviation of the wrist is calculated independent of handedness.

that would be intuitive and easily identified by therapists using palpation techniques. Markers were placed proximal to the joint on the distal head of the proximal bone. In marker placement method 1, markers often move onto the distal segment adjacent to the joint during the grip due to skin movement. This distortion of the resultant vectors does not correspond to the underlying bone/anatomical segment and, therefore, compromises the position of the resultant vector and its relationship to adjoining vectors.

For the purposes of describing the process of the orientation of the vector calculations, each marker was taken into the model as a set of three points indicating the relative $x y$ and $z$ coordinates of the marker in space, relative to the Vicon coordinate system, or the global coordinate system (GCS). A local coordinate system (LCS) is then embedded in the forearm plane defined by the model and subsequent planes and vectors are described relative to the forearm plane. The orientation of movement within the LCS is illustrated in Fig. 1.

\section{B. Movements and Model Calculations}

The movements of the wrist and hand that are generated from the proposed model are defined as flexion/extension and radial/ ulnar deviation of the wrist, flexion/extension of the dorsal aspect of the transverse metacarpal arch, finger flexion/extension at metacarpophalangeal (MCP), proximal interphalangeal (PIP) and distal interphalangeal (DIP) joints, finger abduction/adduction at the MCP, flexion/extension of the MCP and interphalangeal (IP) joints of the thumb, as well as abduction/adduction and rotation through to opposition of the thumb.

The computational model presented in this paper was developed using the MATLAB (MathWorks, Inc., Natick, MA) software package. In instances where the vectors are the only consideration, the scalar product is used to calculate the angle (1), where $\theta$ is the resultant angle for the joint located between two

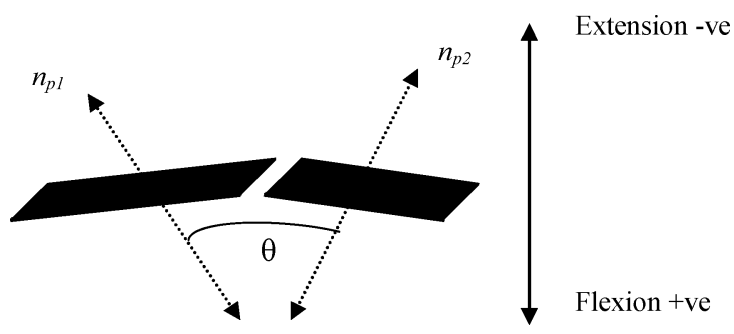

Fig. 2. Flexion/extension between two planes. The direction of the movement remains constant throughout the movements of the metacarpal arch, fingers, and thumb.

segments defined by $\underline{\mathbf{v} 1}$ and $\underline{\mathbf{v} 2}$, where $\underline{\mathbf{v} 1}$ and $\underline{\mathbf{v} 2}$ are two vectors defined from three markers

$$
\theta=\cos ^{-1}[\underline{\underline{\mathbf{v} 1 \bullet \underline{\mathbf{v} 2}}}[\underline{|\mathbf{v} 1||\mathbf{v} 2|}] .
$$

When calculating the angular movement between the two planes of the wrist, a vector normal to each plane is defined. The scalar product between the two normals is calculated to produce the angle for the movement between the two planes. Fig. 2 illustrates this process, where $\hat{\mathbf{n}}$ is a normal vector perpendicular to plane $p$, defined by the cross product of vectors $\underline{\mathbf{v} 1}$ and $\mathbf{v 2}$ (2)

$$
\begin{aligned}
\hat{\mathbf{n}}_{p} & =\underline{\mathbf{v} \mathbf{1}} \times \underline{\mathbf{v} \mathbf{2}} \\
\theta & =\cos ^{-1}\left[\frac{\hat{\mathbf{n}}_{p 1} \bullet \hat{\mathbf{n}}_{p 2}}{\left|\hat{\mathbf{n}}_{p 1}\right|\left|\hat{\mathbf{n}}_{p 2}\right|}\right] .
\end{aligned}
$$

Occasionally, there is a need to reduce a vector into its composite 2-D coordinates, therefore eliminating any secondary out-of-plane movement as discussed in [17]. Projections of a vector onto a plane are calculated using (3), where $\mathbf{v}$ is a vector represented in its composite $\mathbf{x}$ and $\mathbf{y}$ coordinates, projected 
TABLE II

Marker AsSOCiations With Vectors AND Planes

\begin{tabular}{|c|c|c|c|}
\hline Plane/Segment & From & To & Movement Associated \\
\hline \multicolumn{4}{|l|}{ Plane } \\
\hline \multirow[t]{2}{*}{ Forearm } & FAU & WRU & Wrist \\
\hline & FAR & WRR & Wrist \\
\hline \multirow[t]{2}{*}{ Hand } & CMC5 & MCP5 & Wrist \\
\hline & MCP2 & MCP5 & Wrist \\
\hline \multirow[t]{2}{*}{ Radial Hand } & CMCVM & MCP3 & Metacarpal Arch \\
\hline & MCP2 & MCP3 & Metacarpal Arch and part of joint $2-4$ \\
\hline \multirow[t]{2}{*}{ Mid Hand } & CMCVM & MCP4 & Metacarpal Arch \\
\hline & MCP3 & MCP4 & Metacarpal Arch and part of joints $2-4 \& 3-5$ \\
\hline \multirow[t]{2}{*}{ Ulnar Hand } & CMCVM & MCP5 & Metacarpal Arch \\
\hline & MCP4 & MCP5 & Metacarpal Arch and part of joint $3-5$ \\
\hline \multirow[t]{5}{*}{ Thumb } & $\mathrm{CMC} 2$ & MCP2 & Thumb Abd/Add. ${ }^{\dagger} \&$ Rotation \\
\hline & $\mathrm{CMC1}$ & MCP1 & Thumb Abd/Add., Rotation \& F/E \& $^{\S} \mathrm{CP} 1$ \\
\hline & MCP1 & MCP2 & Thumb Abd/Add \& Rotation \\
\hline & MCP1 & IP & F/E MCP1 \\
\hline & IP & FT1 & F/E IP \\
\hline \multicolumn{4}{|l|}{ Segment } \\
\hline Proximal Phalanx & MCP2 & PIP2 & F/E MCP 2nd Finger \& Abd/Add. Fingers 2-3 \\
\hline Middle Phalanx & PIP2 & DIP2 & F/E PIP 2nd Finger \\
\hline Distal Phalanx & DIP2 & FT2 & F/E DIP 2nd Finger \\
\hline Proximal Phalanx & MCP3 & PIP3 & F/E MCP 3rd Finger \& Abd/Add. Fingers 3-4 \\
\hline Middle Phalanx & PIP3 & DIP3 & F/E PIP 3rd Finger \\
\hline Distal Phalanx & DIP3 & FT3 & F/E DIP 3rd Finger \\
\hline Proximal Phalanx & MCP4 & PIP4 & F/E MCP $4^{\text {th }}$ Finger $\&$ Abd/Add. Fingers 4-5 \\
\hline Middle Phalanx & PIP4 & DIP4 & F/E PIP $4^{\text {th }}$ Finger \\
\hline Distal Phalanx & DIP4 & FT4 & F/E DIP 4th Finger \\
\hline Proximal Phalanx & MCP5 & PIP5 & $\mathrm{F} / \mathrm{E} \mathrm{MCP} 5^{\text {th }}$ Finger \\
\hline Middle Phalanx & PIP5 & DIP5 & F/E PIP $5^{\text {th }}$ Finger \\
\hline Distal Phalanx & DIP5 & FT5 & F/E DIP 5th Finger \\
\hline
\end{tabular}

$\uparrow$ Abd/Add $=$ Abduction/Adduction

$\S \mathrm{F} / \mathrm{E}=$ Flexion/Extension

using the scalar product onto the plane $p$ defined by the vectors $\underline{x}$ and $y$

$$
\mathbf{v}_{p}=\mathbf{v}_{\underline{X}}+\mathbf{v}_{\underline{Y}}
$$

where

$$
\begin{aligned}
& \mathbf{v}_{\underline{X}}=(\mathbf{v} \bullet \mathbf{x})_{\underline{X}} \\
& \mathbf{v}_{\underline{Y}}=(\mathbf{v} \bullet \mathbf{y})_{\underline{Y}} .
\end{aligned}
$$

Three equations, therefore, present the foundation for all the model calculations. These principles are applied purely within the context of the specific surface marker placement method defined here for the wrist and hand, which can easily be replicated in a clinical research setting. Table II identifies marker associations with planes and vectors that will be referred to throughout the modeling process.

\section{Wrist Movements}

Vectors were defined from markers placed on the forearm (FAR, FAU, WRR, WRU) and hand (CMC2, CMC5, MCP2, MCP5), which define vectors and planes for those segments, respectively (Fig. 3). Radial/ulnar deviation is calculated from two vectors, one either side of the wrist from markers MCP2 and MCP5 at the hand and WRR \& WRU at the forearm. In this instance both vectors are projected onto the plane defined as the forearm using (3) and the angle calculated using (1). The angle of flexion/extension can be calculated between the normal vectors $\hat{\mathbf{n}}_{p 1}$ and $\hat{\mathbf{n}}_{p 2}$ generated for each plane using (2).

\section{Dorsal Aspect of the Transverse Metacarpal Arch}

The dorsal aspect of the transverse metacarpal arch [18] is described as two joints; $2-4$ indicating the movement between the radial and mid hand planes, and 3-5 indicating the movement between the mid and ulnar hand planes. The numbers in this instance represent the vectors created from metacarpal heads; each vector is then projected onto its constituent plane. A virtual marker CMCVM is created halfway between CMC2 and CMC5. A further three planes are then defined from the position of the virtual marker to the MCP markers 2-5, as illustrated in Fig. 3, which are the radial (RHP), middle (MHP) and ulnar hand planes (UHP). Vectors are defined from adjoining markers placed on the MCP joints, which are first projected onto their corresponding plane using (3) and then the metacarpal arch is defined by calculating the angles between the MCP joints between the RHP and MHP, and also the MHP and UHP using (1). Flexion of the metacarpal arch is defined as the arch of the hand that is created when the fingertips of the fifth finger and thumb move towards the position where the fingertips are touching. Extension is defined when the palm of the hand is moving towards a position where the hand is flat, as when resting on the surface of a desk.

\section{E. Finger Movements}

Three vectors, one for each phalanx are created for each finger using markers placed on the MCP, PIP, DIP, and finger tip. Flexion/extension movements of the MCP, PIP, and DIP joints (2-5) are calculated with respect to the parent hand 

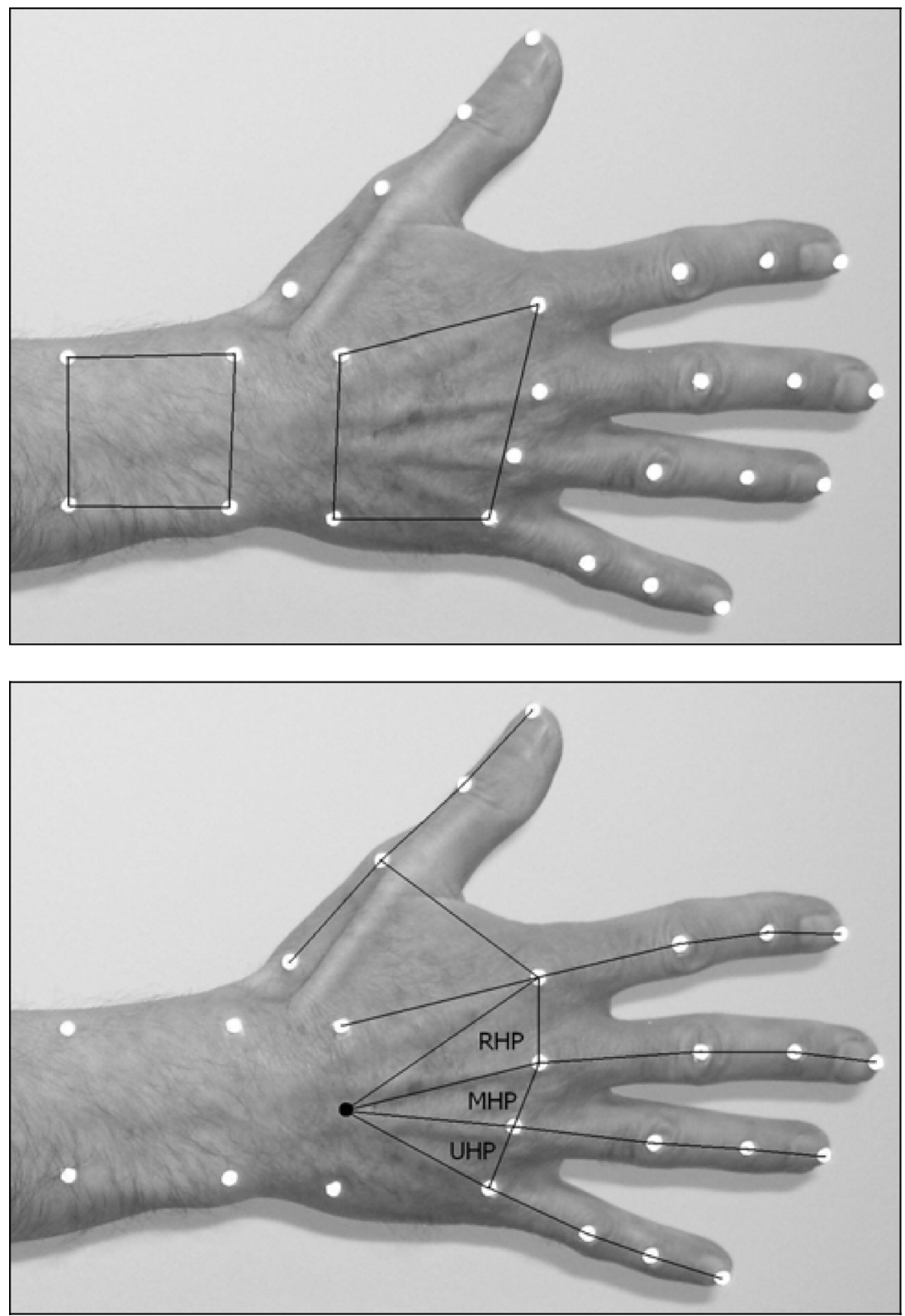

Fig. 3. Vectors and planes generated from markers positioned on the hand and forearm. A virtual marker (CMCVM) is created midway between markers placed at CMC2 and CMC5. CMCVM then joins with the markers placed on the MCP joints to define the radial (RHP), mid (MHP) and ulnar (UHP) hand planes. Vectors are also created for the fingers and thumb.

plane for that vector using (1); where the second finger moved with respect to the RHP, the third and fourth fingers moved respect to the MHP and the fifth finger moved with respect to the UHP (Fig. 3). Finger abduction/adduction is calculated by first projecting the vectors defined for the proximal phalanx of each finger onto the respective hand plane as above, then calculating the resultant angle between the proximal phalanges of each finger to determine the spread of the fingers during object manipulation.

\section{F. Thumb Movements}

Thumb movement is notoriously difficult to define due to the range of movement in all planes [19]-[21]. It is not, therefore, appropriate to represent thumb movement in terms of a single angle of opposition. Thus, in the following method, movement of the thumb is described as a combination of rotation, abd/adduction and flexion/extension of the metacarpophalangeal and interphalangeal joints.

The method to generate angles of thumb movement starts by extending the metacarpal arch to include a thumb plane defined from the first metacarpal and a vector joining MCP1 to MCP2 (Fig. 3). Therefore, normal planes are described from the RHP and thumb plane, and the angle is calculated from the normal vectors using (2). Abduction/adduction of the thumb is calculated from projecting the vectors defined for the first and second metacarpals onto the thumb plane. The angle can then be calculated between the two resultant metacarpal vectors. Thumb flexion/extension of the MCP and IP joints are calculated independently using (1). 


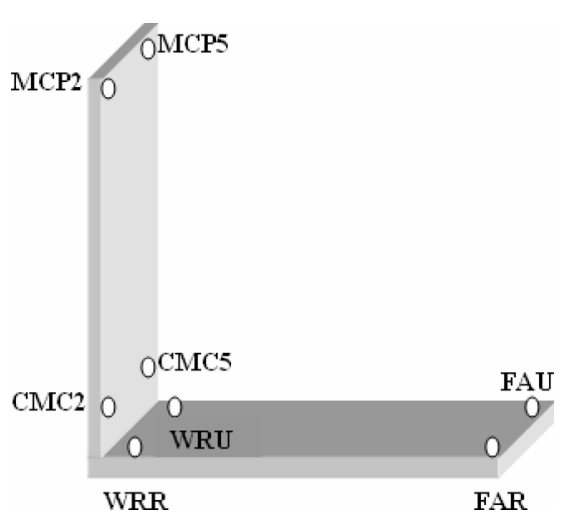

Fig. 4. Metallic reference objects was used for measuring $90^{\circ}$ (extension pictured), $60^{\circ}$, and $30^{\circ}$ angles and comparing against the model output for reliability. For flexion, markers were placed on the opposite face of the objects in the same positions.

\section{MODEL VALIDATION AND PARTICIPANT TRIAL}

\section{A. Static Reference Frames}

The model was validated against thirteen known static reference positions to test the accuracy of the generated output. They were neutral $\left(0^{\circ}\right)$, radial deviation $\left(-90^{\circ},-60^{\circ},-30^{\circ}\right)$, ulnar deviation $\left(+90^{\circ},+60^{\circ},+30^{\circ}\right)$, extension $\left(-90^{\circ},-60^{\circ},-30^{\circ}\right)$ and flexion $\left(+90^{\circ},+60^{\circ},+30^{\circ}\right)$, testing an angle range within and beyond those obtainable in a human participant (Fig. 4). Radial/ulnar deviation and neutral position was measured using a protractor on a flat work surface and flexion/extension was measured using metallic frames precision-made at $90^{\circ}, 60^{\circ}$, and $30^{\circ}$ angles. The marker placement for a small hand and forearm, therefore maximizing the potential error, was replicated on the flat work surface and the face of the metallic frame; thus, thirteen static positions were captured and each repeated ten times.

\section{B. Static Participant Trial-Inter-Rater Reliability}

A study was undertaken to test the repeatability of marker placements between two raters applying markers to a group of unimpaired right handed participants $(n=8$; mean age $=29$, \pm 6 years). The marker placement for the wrist was applied to the dominant wrist and hand of each participant. The time for each rater to apply the markers was between 3 and 5 min. Metallic frames were used on which each participant was asked to place their forearm and hand. The frames provided a series of static positions for the wrist in extension (position A) and two positions of flexion (positions $\mathrm{B}$ and $\mathrm{C}$, respectively). In addition, each participant's forearm and hand was placed on a flat work surface in neutral (position D); radial and ulnar deviation (positions $\mathrm{E}$ and $\mathrm{F}$, respectively) and the resultant positions were captured by the Vicon system.

\section{Dynamic Participant Trial}

Data was collected from unimpaired participants $(n=10$; mean age $=33, \pm 9$ years $)$. Participants were both right $(\mathrm{n}=$ $8)$ and left $(n=2)$ hand dominant. Light and heavyweight spherical objects of the same size from the Southampton Hand

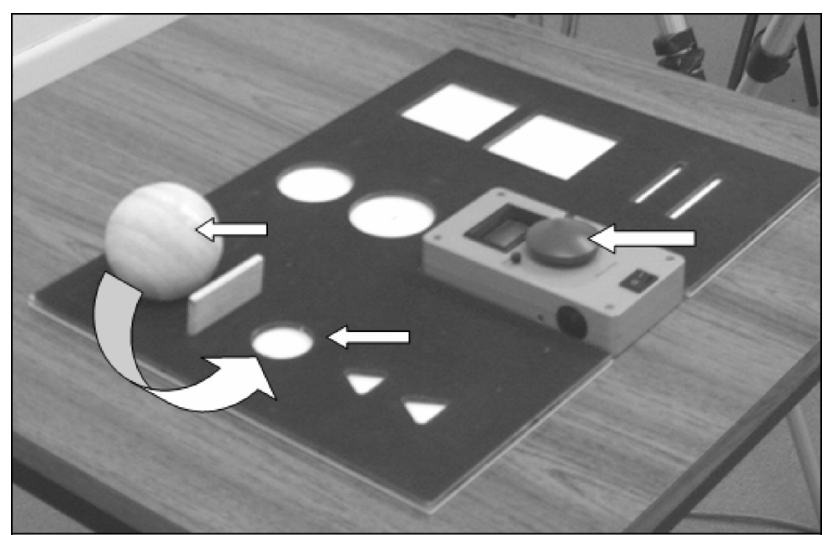

Fig. 5. SHAP spherical object and trial setup for participant repeatability trial. From button press, each participant grasps and lifts the spherical object over the obstacle to the predefined space on the SHAP foam-board, then back to button press to complete the cycle.

Assessment Procedure (SHAP) were used to constrain the gross movement of the wrist and hand, and to provide a recognisably cyclic movement [2], [22]. Each participant sat in a chair with their arms resting on a table with a SHAP form-board placed in front of them. A start/stop position on the SHAP form-board was identified in order to establish a common start and end point for the movement cycle whereby a participant would press their hand to the start/stop position and undertake the task, then replace their hand at the start/stop position on the board. For the task, each participant would grasp and lift a sphere from the furthest to the nearest position on the board and then press their hand back to the start/stop position, indicating the end of the movement cycle (Fig. 5). As part of the task, the sphere must be lifted over a small obstacle $25 \mathrm{~mm}$ high, therefore forcing each participant to lift the weighted object to at least a standard height by clearing the height of the obstacle. Each participant was asked to repeat the lightweight task 5 times, followed by the heavyweight task five times.

\section{RESUlts}

\section{A. Static Reference Frames}

Table III summarizes the results of the ten repeated captures from each of the thirteen static reference positions. The constituent flexion/extension and radial/ulnar deviation offset angles involved in each of the thirteen static positions are summarized as the mean of the ten repeats, the standard deviation and the $95 \%$ confidence interval of the mean for each position. For each primary position of flexion/extension, there will be a component of radial/ulnar deviation which is called the secondary out-of-plane position and similarly for each primary radial/ulnar position there will be a secondary out-of-plane position in flexion/extension. For each primary position, the secondary out-of-plane positions are also presented (shown in italic). The mean angles measured for positions in both primary radial/ulnar deviation and primary flexion/extension are within a degree of the set angle. It should be noted that the angles are set with a protractor which is at best only accurate to a degree, and, thus, the measured angles are within this error margin. The 
TABLE III

Results for the Static Positions Generated From the Model

\begin{tabular}{lcccccc} 
Position & \multicolumn{2}{c}{ Flexion/Extension } & \multicolumn{4}{c}{ Radial/Ulnar Deviation } \\
& Mean & Std. & $95 \%$ & Mean & Std. & $95 \%$ \\
& Angle & Dev. & CI & Angle & Dev. & CI \\
\hline Neutral $0^{\circ}$ & -0.264 & 0.040 & $-0.289--0.239$ & -0.245 & 0.034 & $-0.266--0.223$ \\
Radial $-30^{\circ}$ & 0.135 & 0.042 & $0.109-0.162$ & -29.383 & 0.012 & $-29.391--29.376$ \\
Ulnar $+30^{\circ}$ & -1.387 & 0.076 & $-1.434--1.339$ & 30.309 & 0.278 & $30.133-30.484$ \\
Extension $-30^{\circ}$ & -30.028 & 0.007 & $-30.033--30.024$ & 0.688 & 0.005 & $0.685-0.691$ \\
Flexion $+30^{\circ}$ & 30.056 & 0.005 & $30.053-30.059$ & -0.194 & 0.010 & $-0.200--0.188$ \\
Radial $-60^{\circ}$ & -1.534 & 0.057 & $-1.570--1.497$ & -59.335 & 0.012 & $-59.342--59.327$ \\
Ulnar $+60^{\circ}$ & -1.355 & 0.042 & $-1.381--1.329$ & 60.931 & 0.017 & $60.920-60.941$ \\
Extension $-60^{\circ}$ & -60.496 & 0.003 & $-60.048--60.044$ & -0.993 & 0.025 & $-1.009--0.977$ \\
Flexion $+60^{\circ}$ & 60.185 & 0.004 & $60.182-60.188$ & -0.219 & 0.009 & $-0.225--0.213$ \\
Radial $-90^{\circ}$ & -0.397 & 0.014 & $-0.405--0.388$ & -90.745 & 0.016 & $-90.755--90.735$ \\
Ulnar $+90^{\circ}$ & -0.508 & 0.012 & $-0.516--0.501$ & 90.480 & 0.019 & $90.468-90.493$ \\
Extension $-90^{\circ}$ & -90.402 & 0.014 & $-90.411--90.393$ & 0.564 & 0.011 & $0.557-0.571$ \\
Flexion $+90^{\circ}$ & 89.846 & 0.033 & $89.825-89.867$ & -0.624 & 0.003 & $-0.626--0.622$ \\
\hline
\end{tabular}

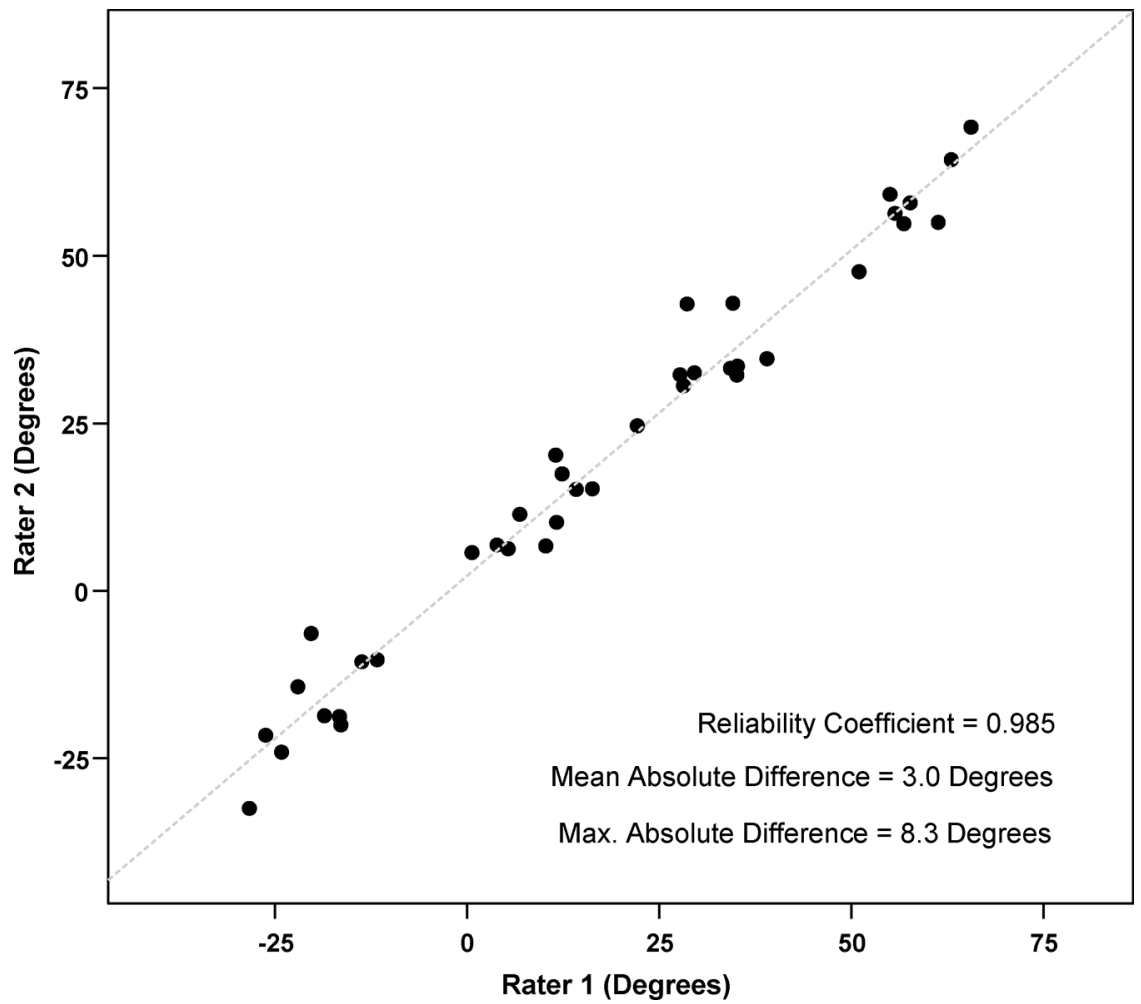

Fig. 6. Relationship of the measured positions A, B, C, and D in flexion/extension defining the marker placement error when markers are independently placed by two raters.

mean angles for the secondary out-of-plane positions are also less than a degree.

The repeatability of the outcome from the model was determined across successive static trials using an intraclass correlation coefficient, which was calculated to be $I C C_{1,1}=0.946$. The results of the measurement accuracy and reliability trial show the model to have high repeatability and to be accurate to within a degree in all directions as is shown in Table III.

\section{B. Static Participant Trial-Inter-Rater Reliability}

Fig. 6 shows the relationship between the measured angles over the four positions A, B, C, and D (flexion/extension) with markers independently placed by two raters. Fig. 7 similarly shows the relationship between the measured angles for the positioned D, E, and F (radial/ulnar deviation) with independently placed markers. In both figures the dotted line shows the line of best fit using a least squares regression.

Both Figs. 6 and 7 clearly demonstrate that there is a correlation between the angles measured by the two raters. The reliability coefficient $(R)$ was calculated as 0.985 for flexion/extension and 0.946 for radial/ulnar deviation (both significant with p-values $<0.001)$. A reliability coefficient greater than 0.75 is considered as giving excellent reliability [23]. The reliability coefficient is calculated as

$$
R=\frac{\sigma_{\text {true }}^{2}}{\sigma_{\text {true }}^{2}+\sigma_{\text {error }}^{2}}
$$




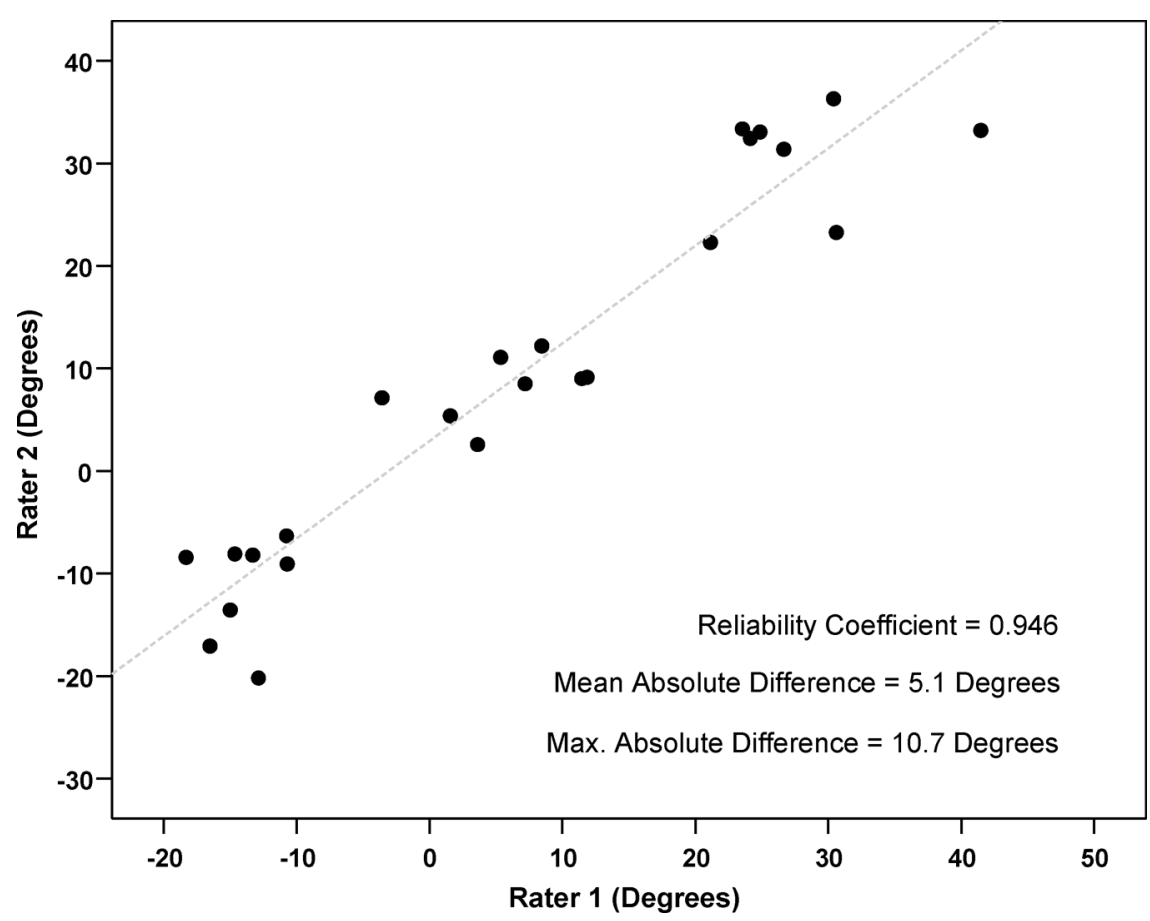

Fig. 7. Relationship of the measured positions $\mathrm{D}, \mathrm{E}$, and $\mathrm{F}$ in radial/ulnar deviation defining the marker placement error when markers are independently placed by two raters.
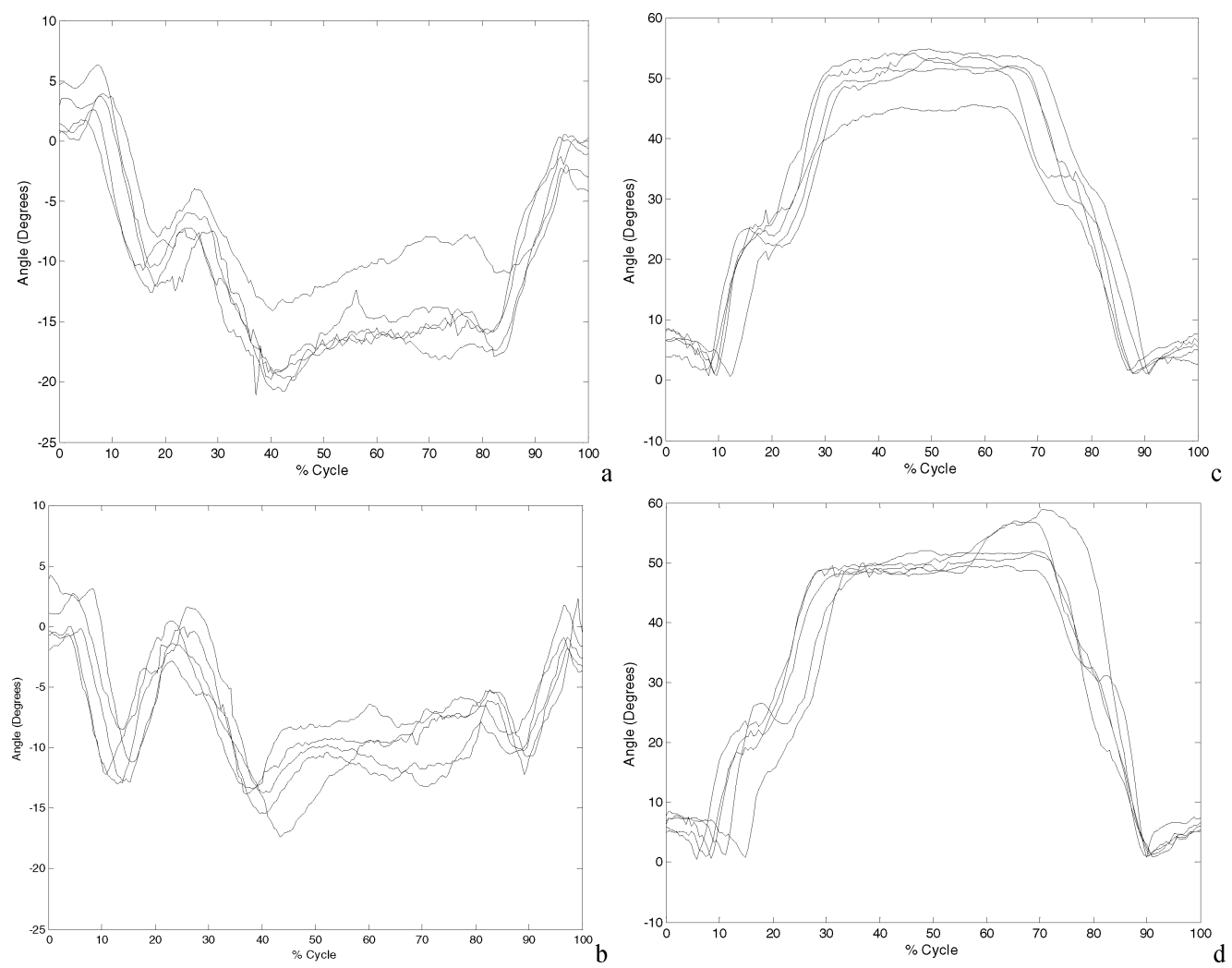

Fig. 8. Wrist and PIP flexion/extension repeats for two sample participants. (a), (b) Five repeats for the wrist during lift of the (a) lightweight and (b) heavyweight objects. (c), (d) Five repeats for the PIP joint of the index finger during lift of the (c) lightweight and (d) heavyweight objects. This illustrates similarities during repeated movements for an individual and a repeated movement strategy by an individual when lifting light and heavyweight versions of an object.

where $\sigma_{\text {true }}^{2}$ is the estimated variance across participants and $\sigma_{\text {error }}^{2}$ is the estimated variance across raters.
For flexion/extension the mean absolute difference between the two raters was $3.0^{\circ}$ with a maximum absolute difference 
TABLE IV

CORRELATION COEFFICIENTS BETWEEN LIGHT AND HEAVy WAVEFORMS FOR AN INDIVIDUAL

\begin{tabular}{lcccccccccc}
\hline \hline Position & P1 & P2 & P3 & P4 & P5 & P6 & P7 & P8 & P9 & P10 \\
\hline Wrist Rad/Uln & 0.697 & 0.981 & 0.900 & 0.065 & 0.943 & 0.859 & 0.475 & 0.857 & 0.637 & 0.403 \\
Wrist Flex/Ext & 0.153 & 0.473 & 0.851 & 0.580 & 0.262 & 0.105 & 0.809 & 0.954 & 0.671 & 0.808 \\
Arch 2 - 4 & 0.893 & 0.929 & 0.859 & 0.756 & 0.960 & 0.674 & 0.390 & 0.772 & 0.713 & 0.589 \\
Arch 3 - 5 & 0.818 & 0.963 & 0.929 & 0.919 & 0.749 & 0.888 & 0.571 & 0.910 & 0.831 & 0.920 \\
MCP2 & 0.942 & 0.936 & 0.722 & 0.651 & 0.986 & 0.361 & 0.874 & 0.598 & 0.898 & 0.890 \\
PIP2 & 0.852 & 0.945 & 0.987 & 0.679 & 0.675 & 0.899 & 0.902 & 0.891 & 0.912 & 0.956 \\
DIP2 & 0.164 & 0.948 & 0.982 & 0.750 & -0.153 & 0.293 & 0.927 & 0.893 & 0.546 & 0.836 \\
\hline \hline
\end{tabular}
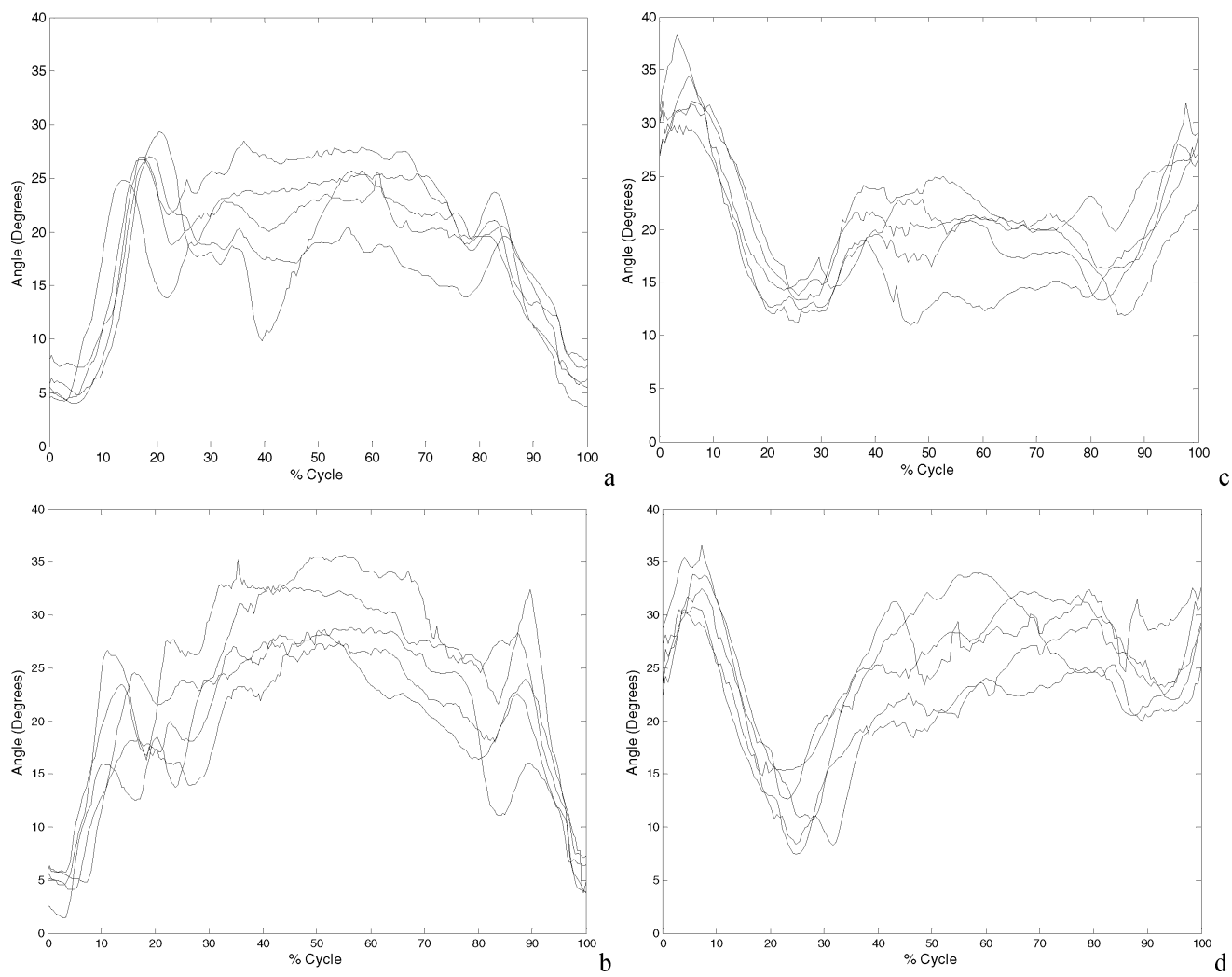

Fig. 9. Flexion/extension of the MCP joint of the index finger for two sample participants illustrating the differences in the movement strategies employed across the population when lifting the objects. (a), (c) Solid lines dedicate lightweight tasks; (b), (d) dashed lines indicate heavyweight tasks.

of $8.3^{\circ}$. For radial/ulnar deviation the mean absolute difference between the two raters was $5.1^{\circ}$ with a maximum absolute difference of $10.7^{\circ}$. In both cases, the mean absolute difference between the two raters is better than that defined in the literature for manual goniometry (between $7^{\circ}$ and $9^{\circ}$ ) which is considered in clinical practice to be the gold standard of joint angle measurement [24], [25].

\section{Dynamic Participant Trial}

The joint angles for the wrist, transverse metacarpal arch and index finger are presented here as an example of the output of the method previously defined in this paper and to illustrate the variability of wrist and hand movement in participants when repeating a movement under constrained conditions. Filtering of the data has been deliberately avoided as there is valuable information in the frequency content of wrist and hand movement.

The waveforms generated from the model output were resampled to $100 \%$ of a cycle to and from the start/stop position on the
SHAP form-board. Fig. 8 illustrates the repeated waveforms for a sample participant for flexion/extension of the wrist and PIP joint of the index finger. Mean waveforms of the five repeats for light and heavy tasks was generated and a Pearson's correlation coefficient was calculated over time between the light and heavy mean waveforms for radial/ulnar deviation and flexion/extension of the wrist, flexion/extension of the metacarpal arch and flexion/extension of the MCP, PIP, and DIP joints of the index finger (Table IV).

On average, participants show a higher correlation in radial/ ulnar deviation $(r=0.65)$ of the wrist when lifting the light and heavyweight spheres compared to flexion/extension $(r=0.44)$. Movement of the metacarpal arch joints showed strong correlations between light and heavy waveforms, where joint 2-4 showed a slightly lower correlation $(r=0.75)$ than joint 3-5 $(r=0.85)$. The waveforms of the MCP and PIP joints of the index finger also showed a strong correlation $(r=0.79$ and $r=0.87$, respectively), while the DIP joint of the index finger 
showed only moderate correlation between light and heavy average waveforms $(r=0.62)$. Although the results of these correlations indicate that an individual uses a similar movement strategy, a common movement strategy, however, was not obvious across the population. Fig. 9 depicts the flexion/extension waveforms for two sample participants when lifting the lightweight and heavyweight objects and illustrates the variability of movement in flexion/extension of the MCP of the index finger across the population. The variability of movement strategies adopted across the population at the MCP joint can also be seen in the wrist, metacarpal arch, PIP, and DIP joints of the index finger.

\section{Discussions AND CONCLUSION}

A marker placement method has been proposed that is intuitive and simple to apply in a clinical research setting. Marker placement methods such as those proposed by Rau et al. [1], Schmidt et al. [13], and Williams et al. [26] are more complex, using triads of markers on each segment. In their study, Rau et al. [1] applied their marker placement method to the measurement of a child with plexus lesion and in this case would be unable to extend their method to include analysis of finger and thumb movements during the patient's compensatory movement strategy. This would be due to the smaller surface area of the fingers, specifically challenging in studies involving children.

Similarly, Fowler et al. [9] use a rod axis system on each segment to denote a plane. If more complex hand movements are to be considered, the number of markers increase by three for each segment analyzed. This is both cumbersome and suffers from problems with marker crossover and occlusion. Also to be considered are certain impairments where spasticity, joint subluxation or deformation would hinder the correct application of the marker set, or are cumbersome for patients to wear in addition to concentrating on functional tasks. Complex marker placement methods may also increase the assessment time needed for a patient to attend a trial and in addition, some methods require static calibration trials to identify intermarker distances and marker triads with respect to markers that were positioned on boney landmarks during static calibrations [1], [10], [13], [15], [26]. Compared to previously published techniques, the proposed marker placement system is simple, using single markers that will not interfere with the movements of the fingers.

In comparison to the examples cited above, the number of markers needed for the proposed marker placement method are kept to a minimum (26 markers), while enabling the simultaneous measurement of all the joints of the wrist and hand. The proposed model is an extension to models previously published by Rash et al. [11] where focus is on the analysis of flexion/extension of an individual finger only, and Small et al. [6] who proposed a model based solely on wrist movements. A similar marker placement method proposed by Carpinella et al. [5] uses only 17 markers but does not provide a method of measuring the wrist angles, metacarpal arch and DIP joints of the fingers as presented in this paper. The metacarpal arch and the curvature it brings to the palm are intrinsic to the opposition of the thumb to the fingers and, therefore, should not be overlooked in models studying the functional movements of the wrist and hand. Although the work of Miyata et al. [3] discusses the metacarpal arch using a proposed system of 25 markers, they do not explicitly provide any model-based calculations to characterize it.

The results of the static reference trial ascertained that the output generated from the model is repeatable and accurate to within one degree in all directions. Differences between repeated captures of a static reference frame were shown to be within a degree of each other, therefore indicating that the output generated from the model can produce repeatable results. The results describe the marker placement as extremely reliable across assessors and accurate to within the limits defined by reliability studies for manual goniometers. Therefore, the marker placement method can be applied in a clinical research setting giving angles with a mean repeatable accuracy of $5.1^{\circ}$. Previously published research has not addressed the issue of reliability of the model output based on marker placement error by additional raters. It was, therefore, not possible to compare these results. Some studies did report the repeated accuracy of their models; Carpinella et al., reported a repeated accuracy of $\pm 7.3^{\circ}$ [5], Degeorges et al., reported a repeated accuracy of $\pm 8.0^{\circ}$ [10], and Dipietro et al., reported a repeated accuracy of $\pm 6.2^{\circ}$ [7]. In addition, Carpinella, et al., also reports a $10-\mathrm{min}$ application time for markers to be placed on participants [5]. The marker placement method proposed in this paper takes between 3 and $5 \mathrm{~min}$ between raters and has been shown to be reliable within this reduced timeframe. The proposed marker placement method and modeling technique are actively being used in ongoing clinical trials to assess functional movements of chronic stroke patients and a splinting intervention for hyperextension of the PIP joint in rheumatoid arthritis.

The model was successfully applied to show that an individual employs a common movement strategy when lifting light and heavy versions of objects. The results from the correlation between the light and heavy lifting tasks indicate a higher correlation in radial/ulnar deviation of the wrist for all participants and this was also illustrated in both joints of the metacarpal arch and the MCP and PIP joints of the index finger (Table IV). Although an individual adopted similar movement strategies to complete related tasks, little conformity could be seen across the population indicating that unimpaired individuals adopt very different lifting strategies when moving the wrist and hand to complete a functional task. The variability shown in the wrist movement may also indicate that the wrist is the final joint of the upper limb that adjusts for the intention of the movement, or functional intention of the task. An individual's idiosyncrasies in prehension may also account for the variability across the population.

The results from the participant repeatability trial can be compared to [14] where wrist angle movements were generated while completing activities of daily living tasks from a SHAP. Movements of jar opening and carton pouring were analysed. Although not reported, during both tasks an individual adopted the same movement strategy to repeat a task, while the movement strategies adopted across the population were shown to be different for the jar opening task. Conformity in movement strategies could be seen across the population for the carton pouring task. This may be due to the prolonged time in which 
the participant maintained a stabilizing grip during the pouring part of the task, whereas the jar opening task is requires a continuous functional movement.

It should be noted that the inter-rater reliability was only performed on the wrist marker placements. Further to this the reliability of the wrist angle is investigated for separate axis at a time. Although some investigation of the coupling of wrist axes, finger joints and metacarpal arch is carried out as part of the dynamic participant trial, future work needs to investigate the reliability of these angles.

The method described in this paper was not developed as a complete biomechanical model and does not produce definitions of skin movement or anatomically correct joint centers, although this could be extended using existing methods for joint center calculations [3]. Although not suitable for use in clinical practice, clinical research would benefit greatly from such an approach. This is evident from the use of gait analysis as a clinical research tool. The method proposed was developed to employ a clearly identifiable and repeatable marker placement from which are calculated the composite movements of the wrist, dorsal aspect of the transverse metacarpal arch, fingers and thumb. It was developed for ascertaining the effectiveness of rehabilitation techniques or quantifying differences in movements during functional activities and can be used as a tool to investigate movement patterns of the wrist and hand in a clinical research setting with minimum interference with the natural movement or impact to the patient.

\section{ACKNOWLEDGMENT}

The authors would like to thank all the individuals who participated in the trials for this research.

\section{REFERENCES}

[1] G. Rau, C. Disselhorst-Klug, and R. Schmidt, "Movement biomechanics goes upwards: From the leg to the arm," J. Biomech., vol. 33, pp. 1207-1216, 2000.

[2] A. Murgia, "A gait analysis approach to the study of upper limb kinematics using activities of daily living," Ph.D. dissertation, University of Reading, U.K., 2005.

[3] N. Miyata, M. Kouchi, T. Kurihara, and M. Mochimaru, "Modelling of human hand link structure from optical motion capture data," in Proc. Int. Conf. Intelligent Robots Systems, Sendai, Japan, 2004, pp. 2129-2135.

[4] F.-C. Su, Y. L. Chou, C. S. Yang, G. T. Lin, and K. N. An, "Movement of finger joints induced by synergistic wrist motion," Clin. Biomech., vol. 20, pp. 491-497, 2005.

[5] I. Carpinella, P. Mazzoleni, M. Rabuffetti, R. Thorsen, and M. Ferrarin, "Experimental protocol for the kinematic analysis of the hand: Definition and repeatability," Gait Posture, vol. 23, pp. 445-454, 2006.

[6] C. F. Small, J. T. Bryant, I. L. Dwosh, P. M. Griffiths, D. R. Pichora, and B. Zee, "Validation of a 3D optoelectronic motion analysis system for the wrist joint," Clin. Biomech., vol. 11, pp. 481-483, 1996.

[7] L. Dipietro, A. M. Sabatini, and P. Dario, "Evaluation of an instrumented glove for hand-movement acquisition," J. Rehabil. Res. Dev., vol. 40, pp. 179-190, 2003.

[8] J. H. Ryu, N. Miyata, M. Kouchi, M. Mochimaru, and K. H. Lee, "Analysis of skin movement with respect to flexional bone motion using MR images of the hand," J. Biomech., vol. 39, pp. 844-852, 2006.

[9] N. K. Fowler and A. C. Nicol, "Functional and biomechanical assessment of the normal and rheumatoid hand," Clin. Biomech., vol. 16, pp. 660-666, 2001.
[10] R. Degeorges, J. Parasie, D. Mitton, N. Imbert, J.-N. Goubier, and F. Lavaste, "Three-dimensional rotations of human three-joint fingers: An optoelectronic measurement. Preliminary results," Surg. Radiol. Anat., vol. 27, pp. 43-50, 2005.

[11] G. S. Rash, P. P. Belliappa, M. P. Wachowiak, N. N. Somia, and A Gupta, "A demonstration of the validity of a 3-D video motion analysis method for measuring finger flexion and extension," J. Biomech., vol. 32, pp. 1337-1341, 1999.

[12] A. Cappello, A. Cappozzo, P. F. La Palombara, L. Lucchetti, and A. Leardini, "Multiple anatomical landmark calibration for optimal bone pose estimation," Human Mov. Sci., vol. 16, pp. 259-274, 1997.

[13] R. Schmidt, C. Disselhorst-Klug, J. Silny, and G. Rau, "A markerbased measurement procedure for unconstrained wrist and elbow motions," J. Biomech., vol. 32, pp. 615-621, 1999.

[14] A. Murgia, P. J. Kyberd, P. H. Chappell, and C. M. Light, "Marker placement to describe the wrist movements during activities of daily living in a cyclical task," Clin. Biomech., vol. 19, pp. 248-254, 2004.

[15] G. Wu, F. C. T. van der Helm, H. E. J. Veeger, M. Makhsous, P. van Roy, C. Anglin, J. Nagels, A. R. Karduna, K. McQuade, X. Wang, F. W. Werner, and B. Buchholz, "ISB recommendation on definitions of joint coordinate systems of various joints for the reporting of human joint motion-Part II: Shoulder, elbow, wrist and hand," J. Biomech., vol. 38, pp. 981-992, 2005.

[16] H.-Y. Chiu, F. C. Su, S.-T. Wang, and H.-Y. Hsu, "The motion analysis system and goniometry of the finger joints," J. Hand. Surg., vol. 23B, pp. 788-791, 1998.

[17] P. L. Cheng and M. Pearcy, "A three-dimensional definition of the flexion/extension and abduction/adduction angles," Med. Bio. Eng. Comp., vol. 37, pp. 440-444, 1999.

[18] S. J. Edwards, D. J. Buckland, and J. D. McCoy-Powlen, Developmental and Functional Hand Grasps. Thorofare, NJ: Slack, 2002.

[19] P. Braido, X. Zhang, R. Hefner, and M. Redden, "A normative database of thumb circumduction in vivo: Center of rotation and range of motion," in Proc. 46th Human Factors Ergonomics Soc., Baltimore, MD, 1999, pp. 1167-1171.

[20] L. Kuo, W. P. Cooney, M. Oyama, K. R. Kaufman, F. Su, and K. An, "Feasibility of using surface markers for assessing motion of the thumb trapeziometacarpal joint," Clin. Biomech., vol. 18, pp. 558-563, 2003.

[21] J.-H. Chang, K.-Y. Ho, and F.-C. Su, "Joint load of the thumb in jar opening," in Proc. 20th Int. Soc. Biomechanics Conf., 2005, p. 117.

[22] C. M. Light, P. H. Chappell, and P. J. Kyberd, "Establishing a standardized clinical assessment tool of pathologic and prosthetic hand function: Normative data, reliability, and validity," Arch. Phys. Med. Rehab., vol. 83, pp. 776-783, 2002.

[23] J. Fleiss, "Reliability of measurement," in The Design and Analysis of Clinical Experiments. New York: Wiley, 1986, pp. 2-32.

[24] B. S. Ellis, A. Bruton, and J. R. Goddard, "Joint angle measurement: A comparitive study of the reliability of goniometry and wire tracing for the hand," Clin. Rehabil., vol. 11, pp. 314-320, 1997.

[25] B. S. Ellis and A. Bruton, "A study to compare the reliability of composite finger flexion with goniometry for measurement of range of motion in the hand," Clin. Rehabil., vol. 16, pp. 562-570, 2002

[26] S. Williams, R. Schmidt, C. Disselhorst-Klug, and G. Rau, "An upper body model for the kinematical analysis of the joint chain of the human arm," J. Biomech., vol. 39, pp. 2419-2429, 2006.

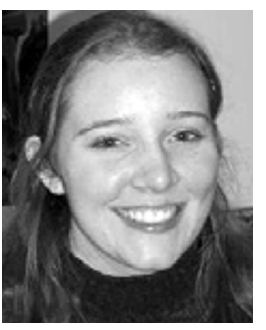

Cheryl D. Metcalf (S'04-M'08) received the B.S degree with honors in computer studies from the University of Sunderland, U.K., in 2002, specializing in adaptive control strategies of robotic grippers, the M.S. degree in evolutionary and adaptive systems from the University of Sussex, U.K., in 2003, specializing in evolving optimized object recognition using haptic sensing, and the Ph.D. degree from the University of Southampton, U.K., in 2007, in kinematic analysis of wrist movements and hand function.

She is currently a Research Fellow at the University of Southampton. Her research interests include the analysis of the effects of motor control deficits and musculoskeletal impairment on hand function and movement strategies of the wrist.

Ms. Metcalf is a member of the IEEE Engineering in Medicine, Biology, and Science Society and a member of the International Society of Biomechanics, where she has just completed a two-year term on the Executive Council. 


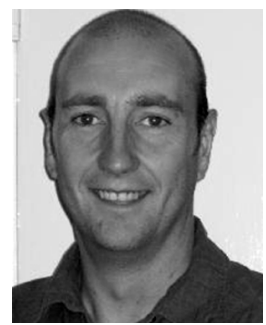

Scott V. Notley received the M.Eng. in information engineering and the Ph.D. degree with a dissertation titled "Prediction of Epileptic Seizures from Depth EEG Recordings" from the University of Southampton, U.K., in 1995 and 2002, respectively.

$\mathrm{He}$ is currently a Research Fellow at the Institute of Sound and Vibration Research (ISVR), University of Southampton. His research interests are nonlinear signal processing and biomedical applications of signal processing, particularly applied to EEG and EMG.

Paul H. Chappell (M'05) graduated with a first-class honors degree in electronics from the University of Sussex, U.K., and received the Ph.D. degree in control engineering from the University of Southampton, U.K.

His research interests are in medical engineering, particularly prosthetics and functional electrical stimulation. He is the author of over 90 publications and is a Senior Lecturer in the School of Electronics and Computer Science, University of Southampton.

Dr. Chappell is a Chartered Engineer and a Member of the IET, the IOP, and the IPEM.

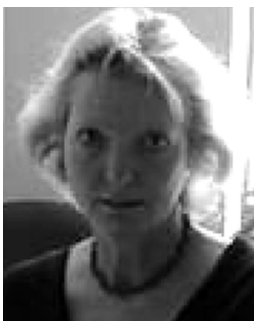

Jane H. Burridge was trained as a Physiotherapist in Bristol, U.K., and as a flautist at the Guildhall School of Music and Drama, U.K. She received the Ph.D. degree in neurorehabilitation from the University of Southampton,U.K.

She leads the multidisciplinary Motor Learning and Control Research program at the University of Southampton. Her specific research interests are in functional electrical stimulation and motor learning and spasticity in neurorehabilitation. Her Ph.D. investigated muscle activation patterns following stroke and response to functional electrical stimulation (FES) to facilitate walking.

Dr. Burridge's current research projects include the use of iterative learning control in robot therapy, motivating mobility using movement feedback games, and a preliminary clinical study using BION microstimulators to facilitate upper limb function in hemiplegia.

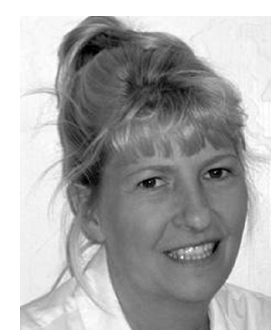

Victoria T. Yule received the B.Sc. degree in sports science from Manchester Metropolitan University, U.K., in 1993, the M.Sc. degree in medical physics and clinical engineering from Sheffield University, U.K., in 1994, and the Ph.D. degree from Salford University, U.K., in 1999.

In 1998, she joined the School of Health Professions and Rehabilitation Sciences, University of Southampton, U.K., where she is involved in both upper and lower limb research using motion analysis. 